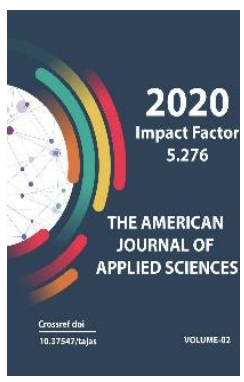

\title{
Classification Of Isoxynoline Alkaloides By Structure And Effect
}

\author{
Xazratqulova I \\ Master's Degree From Fergana State University, Uzbekistan \\ Xazratqulov Z \\ Student Of Fergana Polytechnic Institute, Uzbekistan
}

\section{ABSTRACT}

The chemistry of pyridine and its partially or completely hydrogenated analogues, used in various fields, is one of the fastest growing fields of interest for chemists and pharmacologists. Pyridine, pyridine, and tetrahydroisoquinoline rings are the major fragments of many natural and synthetic biologically active substances. Quaternary pyridinium salts are widely used as alkylating agents, interfacial migration catalysts, antiseptics, ionic liquids as surfactants and anticorrosives.

\section{KEYWORDS}

Isoquinoline and many of its derivatives have different biological activities, stronger base than quinoline

\section{INTRODUCTION}

Isoquinoline has a weak almond odor, a colorless crystalline substance; $\mathrm{tpl}=24.5^{\circ} \mathrm{C}$, tkip $=243^{\circ} \mathrm{C}$. Isoquinoline is well soluble in cold water, organic solvents. It is present in small amounts in the coal seam along with quinoline. Isoquinoline is a stronger base than quinoline.
Upon protonation, isoquinoline forms isoquinoline salts; alkyl, acyl, aryl halides, and dimethyl sulfate to form a rectangular salt. Converted to 1,2,3,4-tetrahydroisoquinoline under water over platinum; with complete water - to cis-decahydroisoquinoline. Oxidation 
by a mixture of ozone and oxygen results in pyridine-3,4-dicarboxylic acid (tsingomeronic acid), which is oxidized to peroxic acid to form isoquinoline-N-oxides. It is known that isoquinoline and many of its derivatives have different biological activities. Some of them are included in various clinical trials or are used in the form of drugs. In addition, the isoquinoline nucleus contains important alkaloids.

In this regard, the introduction of pharmacophore fragments into new isochinoline alkaloids into the aromatic nucleus, methods of their modification, analysis of the obtained substances and the properties of substances obtained by replacing several carbon atoms with nitrogen atoms are of great interest.

To date, isochinoline alkaloids have been well studied and more than a thousand representatives have been isolated from plants. [1-5]. Isoquinoline alkaloids are found in 30 plant families, and almost each of these families has more than 1,000 members. The largest number of them are in the following families: (Annonaceae), barbaris (Berberidaceae), dimyanic (Fumariaceae), gernand (Hernandiaceae), laurel (Lauraceae), magnoli (Magnoliaceae), lunosemyanik (Menispegmaceae), maniapeceae); Papaveraceae), lyutic (Ranunculaceae). In plants, the isoquinoline ring is formed from the amino acids phenylalanine and tyrosine. Isoquinoline alkaloids are divided into the following main groups. Simple isoquinolines (coripalline 1, benzylisoquinolines (papaverine 2), aparfins (gloutsin 3), proaporphins (prouisiferin 4), protoberberins (berbern), benzophenanthridines (sangvinarin5), pavins (argemonin 6), phthalidisoquinolines (phthalidisoxinolines), 2-benzylisoquinolines, 1-phenyl and 4-phenyltetrahydroisoquinolines, azofluorants (rufessin 9), dibenzopyrrocollins (cryptaustoline 10), phenanthrinisoquinolines (morphine 11) and others.

Isoquinoline alkaloid plants have different pharmacological properties. Some of them are widely used in modern and traditional medicine. For example, codeine is an antitussive, morphine is a narcotic analgesic, tubocurarin is a muscle relaxant, emitin is a reversible, antiparasitic, papaverine is an vasodilator and antihypertensive. Most synthetic and semi-synthetic substances are structural modifications of alkaloids designed to alter or enhance the main action properties of the drug, reducing side effects $[6,7]$.

Codeine is a methyl derivative of morphine. The nature of the effect is similar to that of codeine morphine, but it has less analgesic properties and a stronger ability to reduce the central nervous system. It inhibits the activity of the gastrointestinal tract less than morphine. 


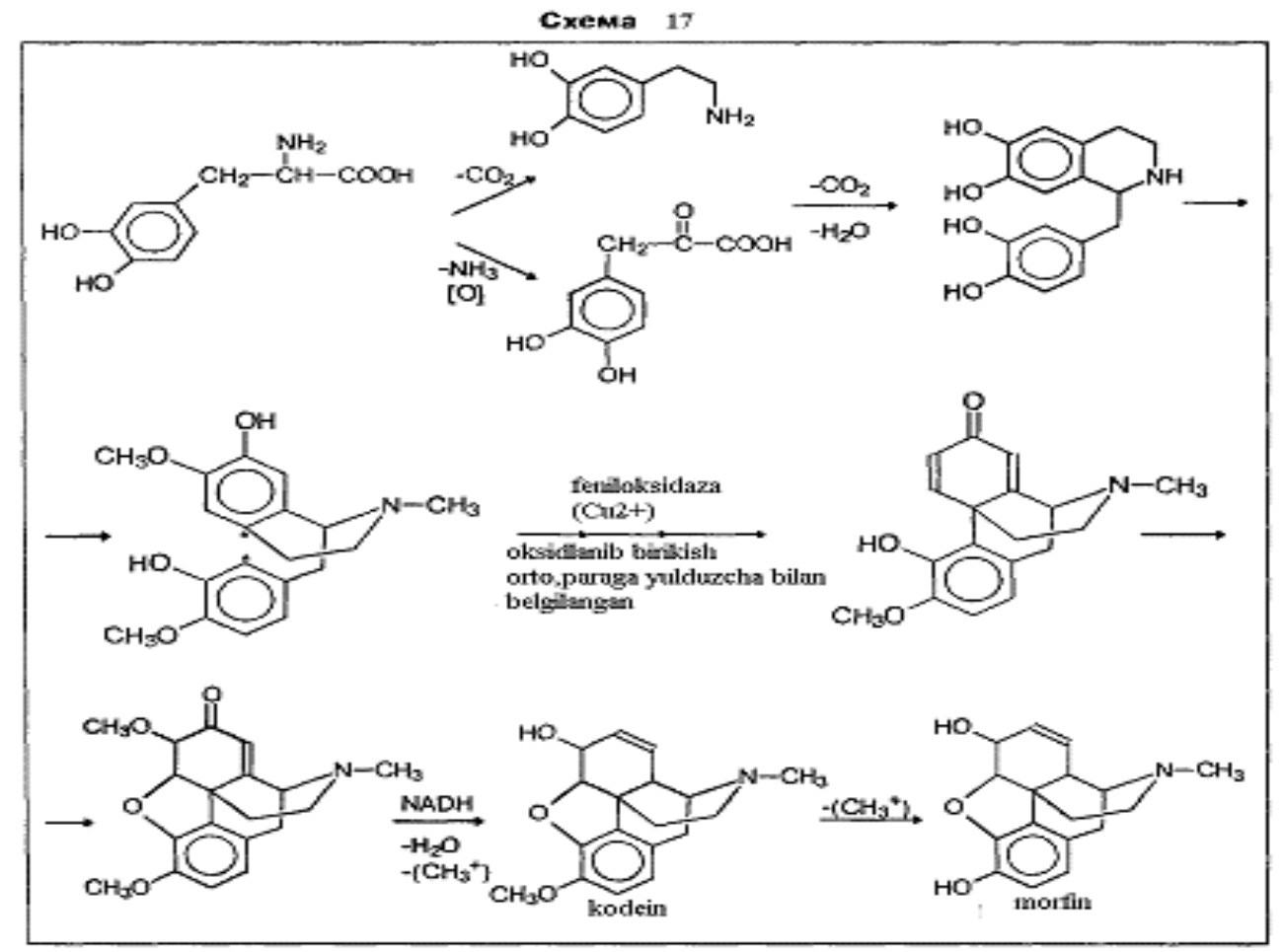

Fig_1

The large number of fundamental reviews and monographs in the field of isoquinoline chemistry suggests that compounds of this class are of great interest [2,3-20]. The study of the chemical and pharmacological properties of such compounds is still relevant today.

Currently, the main focus is on the structural modification and synthesis of isoquinoline derivatives in order to obtain the necessary pharmacological properties. The main directions of the study of the types of activity are directly related to the structure of the compound, and it is more appropriate to study the order of the complexity of the molecular structure. The structural formulas of tyrosine, dopamine, norepinephrine show that they contain a total fragment of phenylethylamine. Numerous studies have been performed on non-metabolized

$1,2,3,4-$ tetrahydroisoquinolines of simple structure involved in dafanenergic processes that affect the activity of the MAT (central nervous system) [63].

Nitrogen compounds of isoquinoline alkaloids form a large group, which includes more than 400 compounds. These compounds have a variety of biological effects, including antibiotic, anti-HIV, antitumor, and antimicrobial activity. Isoquinoline alkaloids are divided into different classes depending on the structure of aporphine, protopins, protoberberines, phthalideisoxinolins, benzophenanthheidins, benzylisoxinolins, morphinans and other spirobenzylisoxinolins [55]. Some of these compounds, due to their biological and pharmacological properties, are widely used in diseases associated with cancer, $A D$ and microorganisms.

\section{CONCLUSION}

Aporphins, benzylisoquinolines, and protoberberins are isoquinoline alkaloids that 
exhibit higher cytotoxic activity than other systemic species. Typically, protoboberberin skeletons have been found in berberine plants (e.g., A549, SK-OV-3, SK-MEL-2, etc.) [56]. Alkaloids from narvidin and encassin were isolated from amarillidaceae.

\section{REFERENCES}

1. DjoulDj., MillsK.//Vkn .: Chemistry of heterocyclic associations.M .: Mir.-2004.SL99.

2. Kuznetsov E. V., Pruchkin D. V., Dorofeenko G. N.// XGS-1974.-№ 2.- P. 181.

3. Bringmann G., Gulder T., Reichert M., Meyer F. Ancisheynine, the First N, Coupled Naphthylisoquinoline Alkaloid: Total Synthesis and Stereochemical Analysis.//Org.Lett.-2006.-V.8.-Nº.-P.10371040.

4. Tolkunov V.S., Shishkin O.V., Zubatyuk R.I., Perepichka I.F., Dulenko V.I. Unusual Double 'O/N to N/O' Recyclization of 5Alkyl-7,8-dimethoxy-2-

aiylbenzo[d]pyrrolo[3,2-b]pyrylium Perchlorates into 5-Alkyl-7,8-dimethoxy-2arylfuro[253-c]isoquinolines.//Synlett.2005.-N6.-P.1036-1038.

5. Achenbach, H., Hemrich, H., 1991. Constituents of tropical medicinal plants. Part 40.Alkaloids, flavonoids and phenylpropanoids of the West African plant Oxymitra velutina. Phytochemistry 30, 1265-1267.

6. Al-Wakeel, S.A.M., Moubasher, M.H., Roberts, M.F., 1995. Alkaloids fromGlaucium corniculatum(L.) of gyptian origin. Biochem. Syst. Ecol. 23, 337-338 